\title{
ON A GRAPH RELATED TO THE MAXIMAL SUBGROUPS OF A GROUP
}

\author{
MARCEL HERZOG ${ }^{\circledR}$, PATRIZIA LONGOBARDI and MERCEDE MAJ
}

(Received 23 July 2009)

Dedicated to the memory of Karl Gruenberg

\begin{abstract}
Let $G$ be a finitely generated group. We investigate the graph $\Gamma M(G)$, whose vertices are the maximal subgroups of $G$ and where two vertices $M_{1}$ and $M_{2}$ are joined by an edge whenever $M_{1} \cap M_{2} \neq 1$. We show that if $G$ is a finite simple group then the graph $\Gamma M(G)$ is connected and its diameter is 62 at most. We also show that if $G$ is a finite group, then $\Gamma M(G)$ either is connected or has at least two vertices and no edges. Finite groups $G$ with a nonconnected graph $\Gamma M(G)$ are classified. They are all solvable groups, and if $G$ is a finite solvable group with a connected graph $\Gamma M(G)$, then the diameter of $\Gamma M(G)$ is at most 2 . In the infinite case, we determine the structure of finitely generated infinite nonsimple groups $G$ with a nonconnected graph $\Gamma M(G)$. In particular, we show that if $G$ is a finitely generated locally graded group with a nonconnected graph $\Gamma M(G)$, then $G$ must be finite.
\end{abstract}

2000 Mathematics subject classification: primary 20E28, 20E32; secondary 20D60.

Keywords and phrases: maximal subgroup, maximal graph, prime graph, solvable groups, simple groups.

\section{Introduction}

In this paper $G$ denotes a nontrivial group. Our aim is to investigate the graph $\Gamma M(G)$ which is associated with the maximal subgroups of $G$. The vertices of $\Gamma M(G)$ are the maximal subgroups of $G$, and we join two distinct vertices $M_{1}, M_{2}$, whenever $M_{1} \cap M_{2} \neq\{1\}$. This graph will be called the maximal graph of $G$. In this paper $p$ and $n$ will always denote primes and positive integers, respectively.

If $G$ is a finitely generated group, then it is easy to show, using Zorn's lemma, that every proper subgroup of $G$ is contained in a maximal subgroup of $G$. Therefore we shall study maximal graphs for finitely generated groups only.

If $G$ is a finite group, then it is easy to check that $\Gamma M(G)$ has exactly one vertex if and only if $G$ is a cyclic $p$-group. Another trivial remark is that the maximal graph of a group $G$ is complete if the Frattini subgroup $\Phi(G)$ is nontrivial. Hence, for example, $\Gamma M(G)$ is complete for all nonelementary abelian finite $p$-groups.

(C) 2010 Australian Mathematical Publishing Association Inc. 0004-9727/2010 \$16.00 
If $G$ is a supersolvable group, then every maximal subgroup of $G$ is of prime index. Therefore, if $G$ is supersolvable, then $\Gamma M(G)$ is complete, except when $G$ is a finite group of order $p q$, where $p, q$ are primes (not necessary distinct). In the latter case, the maximal graph of $G$ has no edges.

Our main aim in this paper is twofold. First, we wish to show that if $G$ is a finite simple group, then $\Gamma M(G)$ is connected and the diameter of $\Gamma M(G)$, denoted by $\operatorname{diam}(\Gamma M(G))$, is bounded. Our bound, 62, for $\operatorname{diam}(\Gamma M(G))$ in the finite simple case is most certainly not the best possible.

We prove the following theorem.

THEOREM 1.1. Let $G$ be a finite simple group. Then:

(1) $\Gamma M(G)$ is connected; and

(2) $\operatorname{diam}(\Gamma M(G)) \leq 62$.

Our second main aim is to determine the finite groups with a nonconnected maximal graph. We prove the following theorem.

THEOREM 1.2. Let $G$ be a finite group. Then the graph $\Gamma M(G)$ is nonconnected if and only if one of the following holds.

(1) $G$ is elementary abelian of order $p^{2}$ ( $p$ a prime).

(2) $G$ is cyclic of order $p q$ ( $p, q$ different primes).

(3) $G=P \rtimes Q$, where $P$ is an elementary abelian $p$-group of order $p^{n}$ ( $p$ a prime), $|Q|=q$, where $q$ is a prime different from $p$, and $Q$ acts irreducibly and fixed point freely on $P$.

Since all groups $G$ described in items (1), (2) and (3) of Theorem 1.2 have edgeless maximal graphs, we obtain the following characterization of finite groups for which $\Gamma M(G)$ has no edges.

Proposition 1.3. Let $G$ be a finite group. Then the graph $\Gamma M(G)$ has no edges if and only if $G$ is solvable and either it is a cyclic p-group or it has the structure described in items (1), (2) and (3) of Theorem 1.2.

Proof. If $\Gamma M(G)$ has no edges, then either it is nonconnected and, by Theorem 1.2, $G$ has the structure described in items (1), (2) and (3) of that theorem, or it is connected, which implies that it has only one vertex and hence, as mentioned above, $G$ is a cyclic $p$-group. The converse is easy to check.

Our first step toward the proof of Theorem 1.2 is the following result. Here $d_{G}\left(M, M_{1}\right)$ denotes the distance in $\Gamma M(G)$ between the maximal subgroups $M$ and $M_{1}$ of $G$ and $d_{G}\left(M, M_{1}\right)>2$ means that either $d_{G}\left(M, M_{1}\right)=n$ for some $n>2$, or $M$ and $M_{1}$ are not connected in $\Gamma M(G)$.

Proposition 1.4. Let $G$ be a finite nonsimple group. Then the graph $\Gamma M(G)$ contains two vertices $M$ and $M_{1}$ with $d_{G}\left(M, M_{1}\right)>2$ if and only if one of the following holds. 
(1) $G$ is elementary abelian of order $p^{2}$ ( $p$ a prime).

(2) $G$ is cyclic of order $p q$ ( $p, q$ different primes).

(3) $G=P \rtimes Q$, where $P$ is an elementary abelian $p$ group of order $p^{n}$ ( $p$ a prime), $|Q|=q, q$ is a prime different from $p$ and $Q$ acts irreducibly and fixed point freely on $P$.

In particular, if the maximal graph of $G$ is nonconnected, then $G$ has the structure described in items (1), (2) and (3).

In view of Proposition 1.4, in order to complete the proof of Theorem 1.2, it is necessary to investigate the connectivity of $\Gamma M(G)$ for finite simple groups. For this purpose, it is useful to consider another related graph, called the prime graph of $G$.

If $G$ is a finite group, then the prime graph $\Pi(G)$ is defined as follows: its vertices are the primes dividing the order of $G$, and two distinct vertices $p$ and $q$ are joined by an edge if and only if $G$ contains an element of order $p q$. This graph was first investigated by Gruenberg and Kegel in an unpublished manuscript [3], and then by many authors (see, for instance, $[1,5,10]$ ). Using their results and those of Sawabe in [8], which rely upon the classification of the finite simple groups, in Section 3 we prove the above stated Theorem 1.1.

Theorem 1.1(1) and Proposition 1.4 together clearly imply Theorem 1.2.

Notice that there exist finitely generated infinite simple groups $G$ with nonconnected graphs $\Gamma M(G)$. Such are, for example, the Tarski monsters, in which all maximal subgroups are of prime order.

We also investigated the diameter of the graph $\Gamma M(G)$ for finite nonsimple groups with a connected maximal graph. It turns out that if $G$ is a finite nonsimple group, then either $\Gamma M(G)$ has no edges or it is connected, with diameter less than or equal to 2. For the exact statement see Theorem 2.1 below.

Section 4 deals with finitely generated groups. In particular, we prove the following Corollary 1.5 , which implies that certain finitely generated generalized solvable groups with a nonconnected maximal graph must be finite.

COROLLARY 1.5. Let $G$ be a finitely generated locally graded group. If $\Gamma M(G)$ is nonconnected, then $G$ is finite.

\section{On the graph $\Gamma M(G)$ for finite nonsimple groups}

Before proving Proposition 1.4, we introduce the appropriate notation. Let $M_{1}, M_{2}$ be vertices of $\Gamma M(G)$. If $M_{1} \cap M_{2} \neq\{1\}$ (allowing $M_{1}=M_{2} \neq\{1\}$ ), then we say that $M_{1}$ and $M_{2}$ are directly connected (always in $\Gamma M(G)$ ) and we write $M_{1} \leftrightarrow M_{2}$. We say that $M_{1}$ and $M_{2}$ are connected, and we write $M_{1} \sim M_{2}$, if they are either equal or there exists in $\Gamma M(G)$ a finite path between them; otherwise they will be called disconnected. A maximal graph will be called nonconnected if it contains two disconnected vertices. Otherwise it will be called connected. If $M_{1} \neq M_{2}$, then the distance function $d_{G}\left(M_{1}, M_{2}\right)$ will denote the length of a shortest path between $M_{1}$ and $M_{2}$ in $\Gamma M(G)$, if such a path exists. If $M_{1}=M_{2}$, then we define $d_{G}\left(M_{1}, M_{2}\right)=0$. 
Thus $d_{G}\left(M_{1}, M_{2}\right) \leq n$ for some $n \in \mathbb{N}$ will mean that either $M_{1}=M_{2}$ or $M_{1} \neq M_{2}$ and they can be connected by a path of length up to $n$. Clearly $d_{G}\left(M_{1}, M_{2}\right)=1$ if and only if $\left\{M_{1}, M_{2}\right\}$ is an edge in $\Gamma M(G)$. If $M_{1}$ and $M_{2}$ are disconnected, then $d_{G}\left(M_{1}, M_{2}\right)=\infty>n$ for all integers $n$. If $\Gamma M(G)$ is connected, then $\operatorname{diam}(\Gamma M(G))$ denotes its diameter.

Proof of Proposition 1.4. We assume, first, that there exist maximal subgroups $M$ and $M_{1}$ in $G$ satisfying $d_{G}\left(M, M_{1}\right)>2$. By our assumptions there exists a proper minimal normal subgroup $N$ of $G$. If $N \cap M \neq\{1\}$ and $N \cap M_{1} \neq\{1\}$, take a maximal subgroup $M_{2}$ of $G$ containing $N$. Then we have $M \leftrightarrow M_{2}, M_{1} \leftrightarrow M_{2}$ and $d\left(M, M_{1}\right) \leq 2$, a contradiction.

Thus, without loss of generality, we may assume that $N \cap M=\{1\}$. Then $G=$ $N \rtimes M$. First we show that $|M|$ is prime. Otherwise, there exists an element $x \in M \backslash\{1\}$ such that $\langle x\rangle<M$. Then $N\langle x\rangle<G$ and there exists a maximal subgroup $M_{3}$ of $G$ such that $N\langle x\rangle \leq M_{3}$. Clearly $M \leftrightarrow M_{3}$. If $M_{1} \cap N \neq\{1\}$, then also $M_{3} \leftrightarrow$ $M_{1}$ and $d_{G}\left(M, M_{1}\right) \leq 2$, a contradiction. If $M_{1} \cap N=\{1\}$, then $G=N \rtimes M_{1}$ and $N\langle x\rangle=N\langle x\rangle \cap\left(N \rtimes M_{1}\right)=N\left(N\langle x\rangle \cap M_{1}\right)$, which implies that $N\langle x\rangle \cap M_{1} \neq\{1\}$. Thus $M_{3} \leftrightarrow M_{1}$ and $d_{G}\left(M, M_{1}\right) \leq 2$, again a contradiction. Therefore $|M|=q, q$ a prime.

Write $M=\langle y\rangle$. If there exists $a \in N \backslash\{1\}$ such that $[a, y]=1$, then $\langle a, y\rangle$ is abelian and $M<\langle a, y\rangle$. Since $M$ is maximal in $G, G=\langle a, y\rangle$ is abelian, $G=\langle a\rangle \times M$ and $a$ is of prime order. It follows that either (1) or (2) holds. If such $a$ does not exist, then $y$ acts fixed point freely on $N$ and $N$ is nilpotent by a theorem of Thompson. Being minimal normal in $G, N$ is an elementary abelian $p$-group for some prime $p$ different from $q$. Moreover, the action of $M$ on $N$ is irreducible, since $M$ is maximal in $G$. Thus (3) holds.

Conversely, if (1), (2) or (3) holds, then $G$ is nonsimple, $\Gamma M(G)$ has at least two vertices, and two different maximal subgroups of $G$ have trivial intersection. So there exist two vertices $M$ and $M_{1}$ of $\Gamma M(G)$ such that $d_{G}\left(M, M_{1}\right)>2$, as required.

It is easy to see that if $G$ is as described in one of the items (1), (2) and (3) of Proposition 1.4, then $\Gamma M(G)$ has no edges.

As a corollary of Proposition 1.4, we obtain the following theorem.

THEOREM 2.1. Let $G$ be a finite nonsimple group. Then one of the following statements holds.

(1) The graph $\Gamma M(G)$ has no edges and $G$ either is cyclic of prime power order or has the structure described in items (1), (2) and (3) of Proposition 1.4.

(2) The graph $\Gamma M(G)$ has at least two vertices and is complete.

(3) The graph $\Gamma M(G)$ has at least two vertices, is connected, $\operatorname{diam}(\Gamma M(G))=2$ and $G$ is a primitive group.

In particular, if $\Gamma M(G)$ is connected, then $\operatorname{diam}(\Gamma M(G)) \leq 2$. 
PROOF. If $\Gamma M(G)$ has only one vertex then, as mentioned above, $G$ is a cyclic group of prime power order and (1) holds. If $\Gamma M(G)$ has two vertices $M$ and $M_{1}$ such that $d_{G}\left(M, M_{1}\right)>2$, then by Proposition 1.4 $\Gamma M(G)$ has no edges and again (1) holds. Otherwise, $\Gamma M(G)$ has at least two vertices, it is connected and $\operatorname{diam}(\Gamma M(G)) \leq 2$. If $\operatorname{diam}(\Gamma M(G))=1$, then (2) holds.

So assume, finally, that $\operatorname{diam}(\Gamma M(G))=2$ and let $M, M_{1}$ be maximal subgroups of $G$ satisfying $d_{G}\left(M, M_{1}\right)=2$. Suppose that $M$ contains a minimal normal subgroup $N$ of $G$. Since $d_{G}\left(M, M_{1}\right)=2$, we have $M \cap M_{1}=\{1\}$ and consequently $M_{1} \cap N=\{1\}$. Hence $G=N \rtimes M_{1}$ and by the maximality of $M$ we have $M=N$ and $\left|M_{1}\right|=q$, a prime. Since $d_{G}\left(M, M_{1}\right)=2$, there exists a maximal subgroup $M_{2}$ of $G$ such that $M_{2} \cap M_{1} \neq\{1\}$ and $M_{2} \cap M \neq\{1\}$. But then $M_{2} \geq M_{1}$, so $M_{2}=M_{1}$ and $M_{2} \cap M=\{1\}$, a contradiction. Hence $M$ contains no minimal normal subgroups of $G$ and $G$ is a primitive group with the stabilizer $M$.

If $G$ is solvable, we can say a little more.

THEOREM 2.2. Let $G$ be a finite solvable group. Then one of the following statements holds.

(1) The graph $\Gamma M(G)$ has no edges and $G$ either is cyclic of prime power order or has the structure described in items (1), (2) and (3) of Proposition 1.4.

(2) The graph $\Gamma M(G)$ has at least two vertices and is complete.

(3) The graph $\Gamma M(G)$ has at least two vertices, it is connected, $\operatorname{diam}(\Gamma M(G))=2$ and $G=N \rtimes M$, where $N$ is the unique minimal normal subgroup of $G$, $C_{G}(N)=N$ and $M$ is a maximal subgroup of $G$ of nonprime order.

In particular, if $\Gamma M(G)$ is connected, then $\operatorname{diam}(\Gamma M(G)) \leq 2$.

PROOF. If $G$ is (abelian) simple, then certainly (1) holds. So assume that $G$ is nonsimple. Then, by Theorem 2.1, one of the items (1), (2) or (3) of Theorem 2.1 holds. Since the groups in items (1), (2) and (3) of Proposition 1.4 are clearly solvable, item (1) of Theorem 2.1 implies item (1) of our theorem. Also item (2) of Theorem 2.1 implies item (2) of our theorem.

So assume, finally, that item (3) of Theorem 2.1 holds. Since $\operatorname{diam}(\Gamma M(G))=2$, there exist two maximal subgroups $M$ and $M_{1}$ of $G$ such that $d_{G}\left(M, M_{1}\right)=2$. Then it follows, as in the last paragraph of the proof of Theorem 2.1, that $\operatorname{Core}_{G}(M)=1$. By the theorem of Baer (see [2, Theorem 15.2]) $G$ has a unique minimal normal subgroup $N$ which satisfies $C_{G}(N)=N$ and $G=N \rtimes M$. If $N$ is a maximal subgroup of $G$, then $M$ is of prime order and $d_{G}(N, M)=2$, which is impossible (see the proof of Theorem 2.1). Thus $N$ is not maximal in $G$ and hence $M$ is not of prime order.

\section{On the graph $\Gamma M(G)$ for finite simple groups}

The main aim of this section is to prove Theorem 1.1. In view of Proposition 1.4, item (1) of Theorem 1.1 also completes the proof of Theorem 1.2. We start with the following basic remark. 
Proposition 3.1. Let $G$ be a finite nonabelian simple group and let $M_{1}, M_{2}$ be maximal subgroups of $G$. Then:

(i) if $\left|M_{1}\right|$ and $\left|M_{2}\right|$ are not coprime, then there exists $g \in G$ such that $M_{1} \sim M_{2}^{g}$ and $d_{G}\left(M_{1}, M_{2}^{g}\right) \leq 2$;

(ii) if $M_{1}$ and $M_{2}$ are of even order, then $M_{1} \sim M_{2}$ and $d_{G}\left(M_{1}, M_{2}\right) \leq 2$.

Proof. (i) Let $p$ be a prime dividing $\left|M_{1}\right|$ and $\left|M_{2}\right|$, and let $a \in M_{1}$ and $b \in M_{2}$, with $|a|=|b|=p$. Moreover, let $P$ be a Sylow $p$-subgroup of $G$ containing $a$. Since $G$ is a nonabelian simple group, we have $P<G$ and there exists a maximal subgroup $M$ of $G$ containing $P$. By the Sylow theorem $b \in P^{h}$ for a suitable $h \in G$. Thus $a \in M \cap M_{1}$ and $b^{h^{-1}} \in M \cap M_{2}^{h^{-1}}$. Therefore $M_{1} \sim M_{2}^{h^{-1}}$ and $d_{G}\left(M_{1}, M_{2}^{h^{-1}}\right) \leq 2$, as required.

(ii) Let $a \in M_{1}$ and $b \in M_{2}$, with $|a|=|b|=2$. Then $\langle a, b\rangle$ is a dihedral group. Thus $\langle a, b\rangle$ is solvable and hence it is a proper subgroup of $G$. Therefore there exists a maximal subgroup $M_{3}$ of $G$ such that $\langle a, b\rangle \leq M_{3}$ and we have $M_{1} \cap M_{3} \neq\{1\}$ and $M_{2} \cap M_{3} \neq\{1\}$. Thus $M_{1} \sim M_{2}$ and $d_{G}\left(M_{1}, M_{2}\right) \leq 2$, as required.

We continue with preliminary results leading to the proof of Theorem 1.1. If $G$ is a finite group, we shall denote the set of all primes dividing $|G|$ by $\pi(G)$. From Proposition 3.1 we obtain the following useful result.

Proposition 3.2. Let $G$ be a finite nonabelian simple group and suppose that $\Gamma M(G)$ is nonconnected. Then there exists a maximal subgroup $M$ of $G$ of odd order such that $M$ is not connected to any maximal subgroup of $G$ of even order.

In particular, $\pi(M) \cap \pi\left(M_{2}\right)$ is empty for any maximal subgroup $M_{2}$ of $G$ of even order.

Moreover, if $p$ is any prime dividing $|M|$ and $P$ is a nontrivial $p$-subgroup of $G$, then $N_{G}(P)$ is of odd order.

Proof. Since $\Gamma M(G)$ is nonconnected, there exist two disconnected maximal subgroups $M$ and $M_{1}$ in $G$. By Proposition 3.1(ii), at least one of them, say $M$, has odd order and if $M$ is connected to a maximal subgroup of $G$ of even order, then $M_{1}$ is also of odd order and is not connected to any maximal subgroup of $G$ of even order. Hence, without loss of generality, we may assume that $M$ is not connected to any maximal subgroup of $G$ of even order. It follows by Proposition 3.1(i) that $\pi(M) \cap \pi\left(M_{2}\right)$ is empty for any maximal subgroup $M_{2}$ of $G$ of even order.

If $p$ divides $|M|$ and $P$ is a nontrivial $p$-subgroup of $G$, then $N_{G}(P)<G$, since $G$ is nonabelian simple. Thus there exists $M_{3}$ maximal in $G$ such that $N_{G}(P) \leq M_{3}$. By Proposition 3.1(i), $M$ is connected to a conjugate of $M_{3}$ and hence $M_{3}$ must be of odd order. In particular, $N_{G}(P)$ is of odd order, as claimed.

Using Proposition 3.2 we obtain another useful result.

Proposition 3.3. Let $G \simeq \operatorname{PSL}\left(2, p^{e}\right)$, where $p$ is a prime and $p^{e}>3$. Then $\Gamma M(G)$ is connected and $\operatorname{diam}(\Gamma M(G)) \leq 3$. 
PROOF. Since $p^{e}>3, G$ is a nonabelian simple group. Let $M$ be a maximal subgroup of $G$ of odd order. If $M$ is a $p$-group, then $|M|=p^{e}$ and $\left|N_{G}(M)\right|=\left(p^{e}-1\right) p^{e} / 2$. By the maximality of $M$, we must have $\left(p^{e}-1\right) / 2=1$, which implies that $p^{e}=3$, a contradiction. Hence $M$ must contain an element $y \neq 1$ of prime order $q$, where $q \neq p$. It follows by Satz II 8.5, Satz II 8.3 and Satz II 8.4 in [4] that $N_{G}(\langle y\rangle)$ has even order. If $M^{*}$ is a maximal subgroup of $G$ containing $N_{G}(\langle y\rangle)$, then it is of even order and $d_{G}\left(M, M^{*}\right) \leq 1$. Since $M$ was an arbitrary maximal subgroup of $G$ of odd order, it follows from Proposition 3.2 that $\Gamma M(G)$ is connected.

Suppose that $M$ and $M_{1}$ are distinct maximal subgroups of $G$. If both are of even order, then $d_{G}\left(M, M_{1}\right) \leq 2$ by Proposition 3.1(ii). So suppose that $M$ is of odd order. As shown above, there exists a maximal subgroup $M^{*}$ of $G$ of even order such that $d_{G}\left(M, M^{*}\right) \leq 1$. If $M_{1}$ is of even order, then $d_{G}\left(M^{*}, M_{1}\right) \leq 2$ and $d_{G}\left(M, M_{1}\right) \leq 3$. So suppose, finally, that both $M$ and $M_{1}$ are of odd order. Then it follows by the list of maximal subgroups of $G$ (see, for example, [9, Example 7, p. 417]) that they are both of order $p^{e}\left(p^{e}-1\right) / 2$. Since $p^{e}>3$, we have

$$
\left[p^{e}\left(p^{e}-1\right) / 2\right]^{2}>\left(p^{e}-1\right) p^{e}\left(p^{e}+1\right) / 2=|G|,
$$

and it follows that $d_{G}\left(M, M_{1}\right)=1$. Thus $\operatorname{diam}(\Gamma M(G)) \leq 3$, and the proof of Proposition 3.3 is complete.

If $G$ is a finite group, then the graph $\Gamma M(G)$ is related to the so-called prime graph $\Pi(G)$, whose vertices are the primes dividing the order of $G$ and two distinct vertices $p$ and $q$ are joined by an edge if and only if $G$ contains an element of order $p q$. In fact we have the following lemma.

LEMMA 3.4. Let $G$ be a finite group, $p$ and $q$ different primes and let $M, M_{1}$ be maximal subgroups of $G$ such that $p$ divides $|M|$ and $q$ divides $\left|M_{1}\right|$. If $p$ and $q$ are connected in the prime graph, then there exists $f \in G$ such that $M \sim M_{1}^{f}$ and $d_{G}\left(M, M_{1}^{f}\right) \leq 20$.

PROOF. Since $p$ and $q$ are connected in $\Pi(G)$, it follows by [5, Theorem 10] that $d_{\Pi(G)}(p, q) \leq 5$. So it suffices to show that if the vertices $p$ and $q$ of $\Pi(G)$ are joined by an edge (that is, there exists an element $x \in G$ of order $p q$ ), then there exists $u \in G$ such that $M \sim M_{1}^{u}$ and $d_{G}\left(M, M_{1}^{u}\right) \leq 4$. Let $a \in M$ be of order $p, b \in M_{1}$ be of order $q$, and let $M_{2}$ be a maximal subgroup of $G$ containing $x$. Then $p$ and $q$ divide the order of $M_{2}$ and, by Proposition 3.1(i), for suitable $g, h \in G$ we have $M \sim M_{2}^{g}, M_{1} \sim M_{2}^{h}$ and $d_{G}\left(M, M_{2}^{g}\right) \leq 2, d_{G}\left(M_{1}, M_{2}^{h}\right) \leq 2$. Hence $M \sim M_{1}^{h^{-1} g}$ and $d_{G}\left(M, M_{1}^{h^{-1} g}\right) \leq 4$, as required.

In [1], Chigira et al. proved, using the classification of finite simple groups, that if $G$ is a finite simple group and $p$ is an odd prime not connected with 2 in the prime graph, then a Sylow $p$-subgroup of $G$ is abelian.

Moreover, Williams proved in [10] that if $G$ is a finite simple group and $\pi$ is a connected component in the prime graph of $G$ not containing 2, then $G$ has a nilpotent 
Hall $\pi$-subgroup $H$ such that, for any $g \in G$, either $H \cap H^{g}=\{1\}$ or $H \cap H^{g}=H$ and $C_{G}(h) \subseteq H$ for each $h \in H \backslash\{1\}$.

Finally, Sawabe recently proved in [8], using the classification of finite simple groups, that if $P$ is an abelian Sylow $p$-subgroup of a finite simple group $G$, then one of the following holds: either $N_{G}(P) / C_{G}(P)$ contains an involution, or $P$ is cyclic, or $G \simeq \operatorname{PSL}\left(2, p^{e}\right)$ for some $p^{e}>3$. Using these results we are able now to prove Theorem 1.1.

Proof of TheOREM 1.1. If $G$ is of prime order, then the theorem certainly holds. By Proposition 3.3, the theorem also holds if $G=\operatorname{PSL}\left(2, p^{n}\right)$. So we may assume that $G$ is a nonabelian simple group and $G \neq \operatorname{PSL}\left(2, p^{n}\right)$. The distance $d_{G}$ in $\Gamma M(G)$ will be denoted by $d$. We shall prove each item separately.

(1) Suppose that $\Gamma M(G)$ is nonconnected. Then, by Proposition 3.2, there exists a maximal subgroup of $G$ of odd order which is not connected to any maximal subgroup of $G$ of even order. Let $q$ be minimal in the set of all primes dividing the order of a maximal subgroup of $G$ not connected to any maximal subgroup of $G$ of even order. Then, by Proposition 3.1(i), no maximal subgroup of $G$ of order divisible by $q$ is connected to a maximal subgroup of $G$ of even order. In particular, each maximal subgroup of $G$ of order divisible by $q$ is of odd order. Moreover, by Lemma 3.4, $q$ is not connected to the prime 2 in the prime graph of $G$.

Let $\pi$ be the connected component in the prime graph of $G$ containing $q$. Then, by the results in [10], there exists a nilpotent $\pi$-Hall subgroup $H$ of $G$ such that, for any $g \in G$, either $H \cap H^{g}=H$ or $H \cap H^{g}=\{1\}$ and $C_{G}(h) \leq H$ for each $h \in H \backslash\{1\}$. There exists $y \in N_{G}(H) \backslash H$, since otherwise it follows from the properties of $H$ that $G$ is a Frobenius group and $G$ is not a simple group, a contradiction. We may assume that $|y|=p$, where $p$ is a prime and $p \neq q$.

Since $H\langle y\rangle<G$, there exists a maximal subgroup $M$ of $G$ containing $H\langle y\rangle$. As $q|| M \mid, M$ is not connected to any maximal subgroup of $G$ of even order and, by the minimality of $q, q<p$. Moreover, $M$ is of odd order and it follows, using the arguments in the proof of Proposition 3.2, that if $r$ is a prime dividing $|M|$ and $R$ is a nontrivial $r$-subgroup of $G$, then $N_{G}(R)$ is of odd order.

Let $Q$ be a Sylow $q$-subgroup of $G, Q \leq H$. Since $y \in N_{G}(H)$ and $H$ is nilpotent, we have $y \in N_{G}(Q)$. Moreover, since $q$ is not connected to 2 in the prime graph of $G$, it follows by [1] that $Q$ is abelian. Hence, by Sawabe's results in [8], either $N_{G}(Q) / C_{G}(Q)$ contains an involution or $Q$ is cyclic or $G \simeq \operatorname{PSL}\left(2, q^{e}\right)$ for some $q^{e}>3$. But $N_{G}(Q)$ is of odd order and, by Proposition 3.3, $G$ is not isomorphic to any such PSL $\left(2, q^{e}\right)$. Hence the only possibility is that $Q$ is cyclic. If $s \in Q$ is of order $q$, then $y \in N_{G}(\langle s\rangle)$ and since $q<p$, it follows that $[s, y]=1$. Consequently $y \in C_{G}(s) \subseteq H$, a final contradiction.

(2) Suppose that the result is false, and there exist maximal subgroups $M$ and $M^{*}$ of $G$ satisfying $d\left(M, M^{*}\right)>62$. Then, by Proposition 3.1(ii), at least one of these subgroups, say $M$, is of odd order. If $d\left(M, M_{e}\right) \leq 30$ for some maximal subgroup $M_{e}$ of $G$ of even order, then $d\left(M^{*}, M_{e}^{*}\right)>30$ for all maximal subgroups $M_{e}^{*}$ of $G$ of even 
order, since otherwise we get, by Proposition 3.1(ii) for some $M_{e}^{*}$,

$$
d\left(M, M^{*}\right) \leq d\left(M, M_{e}\right)+d\left(M_{e}, M_{e}^{*}\right)+d\left(M_{e}^{*}, M^{*}\right) \leq 30+2+30=62,
$$

a contradiction. Therefore we may assume that there exists a maximal subgroup of $G$ of odd order, say $M$, such that $d\left(M, M_{e}\right)>30$ for each maximal subgroup $M_{e}$ of $G$ of even order.

Let $p_{1} \in \pi(M)$. If $p_{1}$ is connected to 2 in $\Pi(G)$, then it follows by Lemma 3.4 that $d\left(M, M_{e}\right) \leq 20$ for some maximal subgroup $M_{e}$ of $G$ of even order, a contradiction. Hence $p_{1}$ is not connected to 2 in $\Pi(G)$.

Let $\pi_{1}$ be the connected component of $\Pi(G)$ containing $p_{1}$. Since $2 \notin \pi_{1}$, it follows by [10], as shown in (1), that there exists a nilpotent Hall $\pi_{1}$-subgroup $H_{1}$ of $G$, such that $C_{G}(h) \leq H_{1}$ for each $h \in H_{1} \backslash\{1\}$ and there exists $y_{2} \in N_{G}\left(H_{1}\right) \backslash H_{1}$ of prime order $p_{2} \notin \pi_{1}$. Moreover, by [1], $H_{1}$ is abelian.

Let $q \in \pi_{1}$ and let $Q$ be a Sylow $q$-subgroup of $H_{1}$. Then $Q$ is abelian. If $N_{G}(Q)$ is of even order, let $M_{e}$ denote a maximal subgroup of $G$ containing $N_{G}(Q)$. Then $M_{e}$ is of even order, and since $p_{1} \in \pi(M), q \in \pi\left(M_{e}\right)$ and $p_{1}, q \in \pi_{1}$, it follows by Lemma 3.4 that $d\left(M, M_{e}^{g}\right) \leq 20$ for some $g \in G$, a contradiction. Hence $N_{G}(Q)$ is of odd order and since $G \neq \operatorname{PSL}\left(2, p^{n}\right)$, it follows by [8] that $Q$ is cyclic. Hence $H_{1}$ is cyclic. Moreover, since $y_{2} \in N_{G}\left(H_{1}\right)$, it normalizes $Q$ and consequently $y_{2}$ normalizes $\langle s\rangle$, where $s$ is an element of order $q$ in $Q$. But $y_{2} \notin H_{1}$, so $\left[y_{2}, s\right] \neq 1$ and since $\left|y_{2}\right|=p_{2}$, a prime, it follows that $p_{2}<q$. Similarly, $p_{2}<r$ for each $r \in \pi_{1}$.

Let $M_{2}$ be a maximal subgroup of $G$ containing $H_{1}\left\langle y_{2}\right\rangle$. Then $p_{1} \in \pi(M) \cap \pi\left(M_{2}\right)$ and it follows by Proposition 3.1(i) that $d\left(M, M_{2}^{g_{2}}\right) \leq 2$ for some $g_{2} \in G$. Clearly $p_{2} \in$ $\pi\left(M_{2}\right)$ and if $p_{2}$ is connected to 2 in $\Pi(G)$, then, by Lemma 3.4, $d\left(M_{2}, M_{e}\right) \leq 20$ for some maximal subgroup $M_{e}$ of $G$ of even order and

$$
d\left(M, M_{e}^{g_{2}}\right) \leq d\left(M, M_{2}^{g_{2}}\right)+d\left(M_{2}^{g_{2}}, M_{e}^{g_{2}}\right) \leq 2+20=22,
$$

a contradiction.

Therefore $p_{2}$ is not connected to 2 in $\Pi(G)$, and if $\pi_{2}$ is the connected component of $\Pi(G)$ containing $p_{2}$, then, arguing as before, we may conclude that there exists a cyclic Hall $\pi_{2}$-subgroup $H_{2}$ in $G$ and an element $y_{3} \in N_{G}\left(H_{2}\right) \backslash H_{2}$ of prime order $p_{3}$, such that $p_{3}<r$ for each $r \in \pi_{2}$. In particular, $p_{3} \notin \pi_{1} \cup \pi_{2}$. Let $M_{3}$ be a maximal subgroup of $G$ containing $H_{2}\left\langle y_{3}\right\rangle$. Then, by Proposition 3.1(i), $d\left(M_{2}, M_{3}^{g_{3}}\right) \leq 2$ for some $g_{3} \in G$ and, as mentioned above, $d\left(M, M_{2}^{g_{2}}\right) \leq 2$. If $p_{3}$ is connected to 2 in $\Pi(G)$, then, by Lemma 3.4, $d\left(M_{3}, M_{e}\right) \leq 20$ for some maximal subgroup $M_{e}$ of $G$ of even order and

$$
\begin{aligned}
d\left(M, M_{e}^{g_{3} g_{2}}\right) & \leq d\left(M, M_{2}^{g_{2}}\right)+d\left(M_{2}^{g_{2}}, M_{3}^{g_{3} g_{2}}\right)+d\left(M_{3}^{g_{3} g_{2}}, M_{e}^{g_{3} g_{2}}\right) \\
& \leq 2+2+20=24
\end{aligned}
$$

a contradiction. Therefore $p_{3}$ is not connected to 2 in $\Pi(G)$.

We continue, by proving the existence of primes

$$
p_{1}>p_{2}>p_{3}>p_{4}>p_{5}>p_{6}
$$


no two of which are connected in $\Pi(G)$ and no $p_{i}$ is connected to 2 in $\Pi(G)$, since otherwise $d\left(M, M_{e}\right) \leq 2(i-1)+20 \leq 30$ for some maximal subgroup $M_{e}$ of $G$ of even order, a contradiction. Hence $\Pi(G)$ has at least seven connected components. But now we have reached the final contradiction, since by [10] the graph $\Pi(G)$ for a finite group $G$ has at most six connected components.

The proof of Theorem 1.1 is complete.

As mentioned above, Theorem 1.2 follows from Proposition 1.4 and item (1) of Theorem 1.1.

\section{On the graph $\Gamma M(G)$ for finitely generated infinite groups}

Our main aim in this final section is to prove the following theorem.

THEOREM 4.1. Let $G$ be a finitely generated infinite nonsimple group. Then the graph $\Gamma M(G)$ is nonconnected if and only if $G=N \rtimes M$, where $N$ is an infinite simple group, $|M|=q, q$ is a prime different from 2 and every nontrivial element of $M$ acts irreducibly and fixed point freely on $N$.

Proof. Assume that the graph $\Gamma M(G)$ is nonconnected and let $M$ and $M_{1}$ be disconnected maximal subgroups of $G$. Let $N$ be a nontrivial proper normal subgroup of $G$ and let $M_{2}$ be a maximal subgroup of $G$ containing $N$. Then either $M_{2} \cap M=\{1\}$ or $M_{2} \cap M_{1}=\{1\}$ and we may assume, without loss of generality, that $N \cap M=\{1\}$. Hence $G=N \rtimes M$ and, arguing as in the proof of Proposition 1.4, we obtain that $|M|=q$, a prime. Let $M=\langle x\rangle$.

First we show that $N$ is a minimal normal subgroup of $G$. In fact, if $N_{1}$ is a nontrivial normal subgroup of $G$ satisfying $N_{1} \leq N$, then $G=N_{1} M=N M$ and since $M \cap N=\{1\}$, it follows that $N_{1}=N$.

Consequently, every nontrivial proper normal subgroup of $G$ is a minimal normal subgroup of $G$ and in particular $G \in \min -n$, that is, $G$ satisfies the minimal condition on normal subgroups.

Next we show that $N$ is an infinite simple group. Since the index $|G / N|=q$ is finite, $N$ must be infinite. It remains only to show that $N$ is a simple group.

Since $G$ satisfies the min- $n$ condition and the index of $N$ in $G$ is finite, it follows by a result of J. Wilson that also $N \in \min -n$ (see, for example, [6, Theorem 5.21]). Let $S$ be a minimal normal subgroup of $N$. Then $S S^{x} S^{x^{2}} \cdots S^{x^{q-1}}$ is normal in $G$, whence $N=S S^{x} S^{x^{2}} \cdots S^{x^{q-1}}$ and $N$ is the direct product of some of the $S^{x^{i}}$ (see [6, Lemma 5.23]). Therefore $S$ is an infinite simple group. In particular, $N$ is nonabelian. Denote by $B$ the set of the $S^{x^{i}}$ involved in the above mentioned direct product.

Now we show that $S=N$.

Assume not. Then, for each $i \in\{1, \ldots, q-1\}, S^{x^{i}} \not \leq S$. Since $S^{x^{j}} \unlhd N$ for $j \in$ $\{1, \ldots, q\}$ and $N$ is a direct product of infinite simple groups, it follows by Remak's theorem (see, for example, [7, Theorem 3.3.12]), that $S^{x^{j}} \in B$ for each such $j$. Thus $N=S \times S^{x} \times S^{x^{2}} \times \cdots \times S^{x^{q-1}}$. Let $a \in S \backslash\{1\}$. Then $D=\left\langle a, a^{x}, \ldots, a^{x^{q-1}}\right\rangle$ is an 
abelian group and $x, a \in N_{G}(D)$. It follows that $N_{G}(D) \geq\langle a, x\rangle>M$ and by the maximality of $M$ we get $N_{G}(D)=G$. Thus $D$ is normal in $G$, contradicting the minimality of $N$. Consequently, $N=S$ is an infinite simple group, as claimed.

Since $M$ is a maximal subgroup of $G$ of prime order, every nontrivial element of $M$ acts irreducibly and fixed point freely on $N$. Finally, $q \neq 2$, since otherwise, letting $a \in N \backslash\{1\}$ and $M=\langle x\rangle$, we get $\left[a, x^{2}\right]=1$, which implies that $[a, x]^{x}=[a, x]^{-1}$. Since $x$ acts irreducibly and fixed point freely on $N$, it follows that $N=\langle[a, x]\rangle$ and $N$ is abelian, again a contradiction.

The converse is obvious.

We conclude by proving Corollary 1.5 .

Proof of Corollary 1.5. Since $G$ is locally graded, each finitely generated subgroup of $G$ has a nontrivial finite homomorphic image. Suppose that $G$ is infinite. Now $G$ is finitely generated, so it also has a nontrivial finite homomorphic image, and since $G$ is infinite, it follows that $G$ is nonsimple.

If $\Gamma M(G)$ is nonconnected, then, by Theorem $4.1, G$ has a normal simple subgroup $N$ of finite index. So $N$ is an infinite finitely generated subgroup of $G$ and does not have a finite nontrivial homomorphic image, a contradiction. Therefore, if $\Gamma M(G)$ is nonconnected, then $G$ must be finite.

\section{Acknowledgement}

The first author is grateful to the Department of Mathematics and Informatics of the University of Salerno for its hospitality and support while this investigation was carried out.

\section{References}

[1] N. Chigira, N. Iiyori and H. Yamaki, 'Nonabelian Sylow subgroups of finite groups of even order', Invent. Math. 139 (2000), 525-539.

[2] K. Doerk and T. Hawkes, Finite Soluble Groups (Walter de Gruyter, Berlin, 1992).

[3] K. W. Gruenberg and O. Kegel, Unpublished manuscript (1975).

[4] B. Huppert, Endliche Gruppen I (Springer, Berlin, 1979).

[5] M. S. Lucido, 'The diameter of the prime graph of a finite group', J. Group Theory 2 (1999), 157-172.

[6] D. J. S. Robinson, Finiteness Conditions and Generalized Soluble Groups, Vol. I (Springer, Berlin, 1972).

[7] D. J. S. Robinson, A Course in the Theory of Groups (Springer, New York, 1982).

[8] M. Sawabe, 'A note on finite simple groups with abelian Sylow p-subgroups', Tokyo J. Math. 30 (2007), 293-304.

[9] M. Suzuki, Group Theory I (Springer, Berlin, 1982).

[10] J. S. Williams, 'Prime graph components of finite groups', J. Algebra 69 (1981), 487-513.

MARCEL HERZOG, School of Mathematical Sciences, Raymond and Beverly Sackler Faculty of Exact Sciences, Tel-Aviv University, Tel-Aviv, Israel e-mail: herzogm@post.tau.ac.il 
PATRIZIA LONGOBARDI, Dipartimento di Matematica e Informatica, Università di Salerno, via Ponte don Melillo, 84084 Fisciano (Salerno), Italy e-mail: plongobardi@unisa.it

MERCEDE MAJ, Dipartimento di Matematica e Informatica, Università di Salerno, via Ponte don Melillo, 84084 Fisciano (Salerno), Italy e-mail: mmaj@unisa.it 